\title{
Anabases
}

ANABASES Traditions et réceptions de l'Antiquité

$22 \mid 2015$

Varia

\section{Les traités normatifs brāhmaniques dans le comparatisme des mariages indo-européens de $\mathrm{G}$. Dumézil}

\section{Guillaume Ducœur}

\section{(2) OpenEdition \\ Journals}

Édition électronique

URL : http://journals.openedition.org/anabases/5410

DOI : $10.4000 /$ anabases. 5410

ISSN : 2256-9421

Éditeur

E.R.A.S.M.E.

\section{Édition imprimée}

Date de publication : 20 octobre 2015

Pagination : $27-48$

ISSN : $1774-4296$

\section{Référence électronique}

Guillaume Ducœur, "Les traités normatifs brāhmaniques dans le comparatisme des mariages indoeuropéens de G. Dumézil », Anabases [En ligne], 22 | 2015, mis en ligne le 20 octobre 2018, consulté le 21 octobre 2019. URL : http://journals.openedition.org/anabases/5410; DOI : 10.4000/anabases. 5410 


\section{Les traités normatifs brāhmaniques dans le comparatisme des mariages indo-européens de G. Dumézil}

Guillaume Duccur

$M$ algré plus de deux siècles de reconstructions du vocabulaire indo-européen, les linguistes n'ont pu restituer un terme équivalent au français "mariage », abstrait dérivé de la forme verbale "marier », elle-même issue du verbe latin maritare. Si la terminologie indo-européenne liée à la parenté,

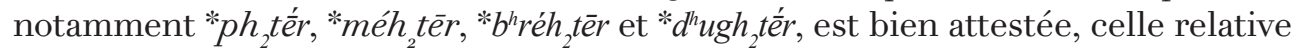
à la conjugalité témoigne non seulement d'une plus grande diversité lexicale, mais encore d'une particularité morphologique pour laquelle des formes verbales et nominales se répartissent respectivement entre les gentes masculine et féminine' Ainsi, le verbe le mieux restituable demeure ${ }^{*} h_{2} u e d h$ - pour signifier qu'un homme conduit une femme à sa maison, c'est-à-dire qu'il l'épouse, après que le père la lui a donnée $\left({ }^{*} d \bar{o}-\right)$. La mariée est donc celle qui a été conduite dans la maison de son époux comme le confirme le sanskrit védique $v a d h \bar{u}^{2}$. Cette reconstruction du mode d'union indo-européen à partir d'un vocabulaire commun ne peut

1 Sur ce champ sémantique indo-européen voir É.Benveniste, Le vocabulaire des institutions indo-européennes. I. Économie, parenté, société, Paris, Les éditions de Minuit, I969, p. 239-244; T. V. Gamkrelidze and V. V. Ivanov, Indo-European and the IndoEuropeans: a Reconstruction and Historical Analysis of a Proto-Language and a ProtoCulture, Berlin, Mouton de Gruyter, I994, p.658-66o; J.P.Mallory and D. Q. Adams, Encyclopedia of Indo-European Culture, Chicago, Fitzroy Dearborn Publishers, I997, p.369-37o; B.W.Fontson, Indo-European Language and Culture: an Introduction, Oxford, Blackwell Publishing, 20I0, p. 2I.

2 En sanskrit classique, l'un des termes employés pour dire qu'un homme épouse une femme est vivāha (<vi- $\sqrt{ }$ vah-, « emmener [une femme chez soi]»). 
évidemment pas refléter l'ensemble des pratiques matrimoniales qui ont pu être en usage parmi les différents peuples de la sphère linguistique indo-européenne. Elle permet uniquement de concevoir qu'un certain type de mariage, qui subsista de manière récurrente parmi différentes cultures héritières de la langue proto-indoeuropéenne, consistait au don d'une jeune femme par son père à un homme qui l'emmenait alors dans sa propre famille ${ }^{3}$. En cela, la sphère indo-européenne ne dénote d'aucune spécificité. Aussi, les études actuelles qui tentent de mieux saisir la structure familiale proto-indo-européenne, investissent de nouveaux champs anthropologiques fondés sur la recherche phylogénétique. En 20II, Laura Fortunato de l'University College London a ainsi montré que la monogamie avait été le principal mode matrimonial proto-indo-européen et que la polygynie ou polygamie n'avait été qu'une évolution possible en fonction de facteurs historiques particuliers ${ }^{4}$. De son côté, Sergey Kullanda de l'Académie des Sciences de Russie a renouvelé la reconstruction de l'organisation sociale proto-indo-européenne par l'hypothèse d'une société tripartite fondée sur des tranches d'âge précises qui auraient contribué par la suite à l'émergence du système social indo-iranien des varna/pištra ${ }^{5}$. Une fois mariés, les jeunes hommes, qui, à l'âge de quinze ou seize

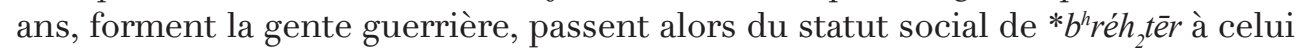
de *ph t tếr tout comme les jeunes mariées du statut social de * $d^{h} u g h_{2}$ tếr à celui de *méh $h_{2}$ têr. L'archéologie des mariages indo-européens est donc loin d'être terminée. Elle s'est ouverte ces dernières années sur des approches linguistiques, anthropologiques, sociales et phylogénétiques qui renouvellent les anciennes explications proposées au cours des $\mathrm{xIX}^{\mathrm{e}}$ et $\mathrm{xx}^{\mathrm{e}}$ siècles.

3 La terminologie se rapportant à l'idée de “mariage» est propre à chaque culture. En tibétain, le terme $\mathbf{5} \mathbf{N}$ (chang sa) désigne le fait de se réunir pour boire ensemble, notamment lors de l'union matrimoniale d'un homme et d'une femme. En chinois classique, 婚姻 (hūn yīn) signifie aussi bien “se marier» que “mariage». Les idéogrammes renvoient, pour 婚 (hūn, femme/crépuscule), au fait que les mariages étaient célébrés le soir, et, pour 姻 (yīn, femme-siège), à l'idée qu'une femme mariée habitait dans la maison de son mari et qu'elle lui était attachée. Chez les Hmong blancs du Laos, le verbe sib yuav, qui signifie le fait pour un homme de prendre une femme afin qu'elle lui appartienne, peut également être plus explicite: sib yuav ua niam txiv, le fait pour un homme de prendre une femme afin qu'ils deviennent épouse et époux, c'est-à-dire mère (niam) et père (txiv). Les formes verbale et nominale de l'hébreu לעב renvoient, quant à eux, à l'homme qui domine ou qui est le maître de la femme, etc.

4 L. Fortunato, “Reconstructing the History of Marriage Strategies in Indo-EuropeanSpeaking Societies : Monogamy and Polygyny ", Human Biology 83/1, 20II, p. 87-Io5.

5 S. Kullanda, "Early Indo-European social organization and the Indo-European homeland „, Journal of Language Relationship 9, 2013, p. 137-I44. 
Sans revenir sur les recherches des érudits européens du XIX $^{\mathrm{e}}$ siècle, il convient de signaler que, par leur présence coloniale en Inde même, les administrateurs britanniques s'intéressèrent de près aux traités normatifs brāhmaniques. Certains savants étudièrent les modes de mariages indiens tels le juriste anglais John D. Mayne (I828-I9I7) dans son volumineux ouvrage sur les lois indiennes ${ }^{6}$, l'indianiste britannique Arthur A. MacDonell (I854-I93o) et le juriste écossais A. B. Keith (I879-1944) dans leurs études védiques. Ces derniers y consacrèrent notamment un substantiel article à l'entrée pati (<*pótis, époux) et patnī (<*pótnī, épouse) au sujet des formes de mariage, de la polygamie, de la polyandrie, du remariage et du veuvage en Inde védique 7 . L'indianiste français Émile Sénart (I847-I928) aborda également dans une perspective comparative indo-européenne l'implication des mariages dans le fonctionnement du système des castes indiennes ${ }^{8}$. Aussi, la liste exhaustive des indianistes européens comme des savants indiens eux-mêmes qui investirent l'ensemble des textes religieux et normatifs de la littérature védique et post-védique relative aux formes de mariages dont témoigne la longue histoire de la civilisation indienne, représenterait aujourd'hui un important travail de recherche.

Parallèlement à ces études de droit indien ancien, la grammaire et la mythologie comparées indo-européennes prirent leur essor en Europe. Or, les principaux comparants dans ces deux domaines d'étude furent incontestablement le sanskrit et les sources textuelles indiennes. Dans la quête identitaire européenne d'une origine et d'un passé indépendants de la tradition judéo-chrétienne, la littérature sanskrite fut alors considérée comme le réservoir immuable et anhistorique d'une tradition religieuse qui serait parvenue à conserver son héritage indoeuropéen grâce à une transmission orale infaillible perpétuée au sein des écoles brāhmaniques. Si du côté européen, Max Müller (I823-ıgoo) pouvait affirmer que “dans la mesure où nous sommes ārya par la langue, c'est-à-dire, par la pensée, jusqu'à présent le Rg veda est notre plus ancien livre» (In so far as we are Aryans in speech, that is, in thought, so far the Rg Veda is our own oldest book ${ }^{9}$ ), du côté indien, l'historien et nationaliste hindou Bal Gangadhar Tilak (I856-I920) déclarait qu'au sujet de la période dite pré-Orion, de 6000 à 4000 av. J.-C., seuls les Indo-ārya, à la différence des Grecs et des Iraniens, avaient conservé, dans leur composition des hymnes ṛvédiques, des souvenirs sur ces temps reculés, et avaient « conservé toutes

6 J. D. Mayne, A Treatise on Hindu Law and Usage, 3rd edition, revised and enlarged, Madras, Higginbotham, I883.

7 A. A. MacDonell and A. B. Keith, Vedic Index of Names and Subjects, vol. I, London, John Murray, I9I2, p. 474-489.

8 É. SÉnARt, Les castes dans l'Inde. Les faits et le système, Paris, E. Leroux, I896.

9 A. KAEGI, The Rigveda: the Oldest Literature of the Indians, Boston, Ginn and Company, I886, p. 25 . 
les traditions avec une fidélité ultrareligieuse et un esprit scrupuleux» (They have preserved all the traditions with a super-religious fidelity and scrupulousness $\left.{ }^{10}\right)$. Le philosophe et historien Sarvepalli Radhakrishnan "1 (I888-I975), président de l'Inde en rg62, s'opposait fermement, quant à lui, au concept d'une Inde éternelle. En I926, face à son auditoire du Manchester College d'Oxford, il rappela que «l'idée que dans l'Inde le temps est demeuré immuable pendant des siècles sans nombre, et que rien n’a changé depuis que la mer primordiale s'est desséchée, cette idée est fausse d'outre en outre. [...] Jamais il n'y eut rien de tel qu'un hindouisme uniforme, stationnaire, immuable, sur quelque point que ce fût de croyance ou de pratique $^{12}$ ». Aussi reconnaissait-il que l'indo-āryanité relevait de la pure idéologie. L'histoire de l'Inde prouvait, en effet, que les différents peuples ārya et anārya, qui avaient vécu sur le sol du sous-continent, en vinrent inévitablement à se mêler au cours des $\mathrm{II}^{\mathrm{e}}$ et $\mathrm{I}^{\mathrm{er}}$ millénaires avant notre ère, tout comme ils se mêlèrent de nouveau avec les différents peuples qui franchirent successivement les frontières du Nord-Ouest indien au cours des millénaires suivants. Si les auteurs des traités normatifs brāhmaniques avaient prôné, en leur temps, un certain type d'unions matrimoniales, S. Radhakrishnan avait bien conscience que le paysage conjugal de l'Inde avait été historiquement bien plus complexe:

“Alors que Manou ${ }^{13}$ recommande les mariages entre membres de même caste (savarṇa), il tolère seulement les mariages d'hommes avec des femmes de castes inférieures (anuloma). Bien qu'il ne justifie pas les mariages pratiloma, c'est-à-dire entre femme de haute caste et homme de caste inférieure, il décrit les deux modes de progéniture issus de telles unions. Quoiqu'elles ne fussent pas approuvées, sans aucun doute elles furent nombreuses. Les castes de type mixte ont été prônées pour régulariser la situation de groupes originairement produits par des mariages interdits ou désapprouvés soit par la loi, soit par la coutume, mais admis après un certain temps. Certains des groupes aujourd'hui considérés comme "intouchables" sont issus, dit-on, d'unions irrégulières ${ }^{14}$. ’

10 B. G. Tilak, The Orion or Researches into the Antiquity of the Vedas, Bombay, Radhabai Atmaram Sagoon, I893, p. 206.

1 Sur l'approche historique de S. Radhakrishnan voir G. Duccur, "Histoire comparée des religions et construction identitaire nationaliste dans le processus d'indépendance de l'Inde», in Th. Di Costanzo et G.Duccur (éd.), Decolonization and the Struggle for National Liberation in India (Igog-I97I), Historical, Political, Economic, Religious and Architectural Aspects, Berne, Peter Lang, 2014, p. 95-129.

12 S. Radhakrishnan, L'hindouisme et la vie, Paris, F. Alcan, I935, p. I33-i34.

13 Manu, ancêtre mythique des Indo-ārya, fut l'auteur, selon la tradition brāhmanique, d'un traité normatif, le Mānavadharmaśāstra, principale source textuelle utilisée par G.Dumézil dans son étude sur les mariages indo-européens.

14 RadHaKrishnan, L'hindouisme, p. IOo. 
Il apparaît donc bien délicat, d'un point de vue historique, de restituer des formes de mariages proprement indo-ārya, et par conséquent proto-indo-européennes, là où les pratiques matrimoniales des traités normatifs brāhmaniques ne remontent guère au-delà $d u v{ }^{\mathrm{e}} \mathrm{s}$. av. J.-C. et peuvent donc avoir été, pour certaines, des modes de mariages anārya partiellement ou totalement indo-āryanisés. Néanmoins, les écoles philologiques allemandes qui avaient travaillé sur les corrélations entre langage et pensée, les indianistes qui pensaient que l'Inde avait su préserver à travers les millénaires ses croyances archaïques, les grammairiens qui restituaient progressivement une langue proto-indo-européenne unitaire, les mythologues qui reconstruisaient des mythèmes communs se plurent à accorder à l'Inde une primauté certaine, celle de l'ancienneté et du conservatisme. Il n’est donc pas surprenant, en un tel contexte scientifique, que Georges Dumézil (I898-I986), ait pris comme comparant primordial, au moins depuis ses premières recherches comparatives sur les boissons d'immortalité ${ }^{15}$ en I924, l'Inde et sa littérature mythologique. Notre contribution sur l'archéologie des mariages indoeuropéens a donc pour finalité de montrer comment G. Dumézil, pris dans la sociologie et le structuralisme de son époque, appliqua, à partir de I952, sa théorie de la trifonctionnalité indo-européenne aux différentes formes de mariages énumérées et classifiées dans le Mānavadharmaśāstra (MnŚ). Nous verrons alors que l'Inde était pour le savant français un monolithe sans histoire qui, en tant que comparant, lui offrait constamment l'opportunité d'étayer et de consolider sa théorie trifonctionnelle indo-européenne qu'il y avait puisé dès I938. La relecture de sa théorie des mariages indo-européens montrera la fragilité de son approche philologique et historique des sources indiennes et l'impartialité de sa méthode comparée trop souvent réductrice et déformatrice. Elle devrait permettre aux spécialistes de Rome de réinvestir le dossier des mariages romains et de proposer une nouvelle approche que celle de l'idéologie trifonctionnelle indo-européenne que G. Dumézil avait tentée en son temps.

En 1979, dans son ouvrage Mariages indo-européens suivi de quinze questions romaines $^{16}$, G. Dumézil consacra une centaine de pages à l'exposition d'une synthèse sur ses recherches concernant les modes de mariages indo-européens débutées en I943. Il présenta en dix pages la restitution tripartite des modes de

15 G. DumÉziL, Le festin d'immortalité, étude de mythologie comparée indo-européenne, Paris, P. Geuthner, r924. Dès sa thèse, G. Dumézil utilisa les sources indiennes comme comparants face aux autres textes appartenant aux cultures de langues indo-européennes, pour opérer ensuite un renversement et en faire des comparés. Ceci est dû au fait que le mythologue français considérait les cycles mythologiques indiens comme les mieux conservés et les plus complets.

16 G. Dumézil, Mariages indo-européens suivi de quinze questions romaines, Paris, Payot, I979, p. I7-II8. 
mariages indiens à partir de laquelle toute la démonstration relative aux formes de mariages à Rome et à l'héritage matrimonial indo-européen fut faite. Plus élogieux que critiques, les comptes rendus rédigés surtout par des spécialistes de la Rome antique ${ }^{17}$ et les reprises postérieures de la théorie, dans d'autres ouvrages ${ }^{18}$, sont éloquents. Ils montrent qu'aucun de ces lecteurs ne s'est donné la peine de suivre la démonstration de G.Dumézil en lisant les sources sanskrites en regard. Aucun ne s'est intéressé à la pertinence et de ses traductions et de son comparatisme. G.Dumézil réitéra, en effet, la même approche comparative que celle qu'il avait développée depuis sa thèse doctorale. L'idée maîtresse, qu'il hérita des linguistes et mythologues du XIx ${ }^{\mathrm{e}}$ siècle, était d'admettre que toute culture qui avait conservé des éléments lexicaux et morphologiques proto-indo-européens avait dû assurément hériter d'une pensée commune également proto-indo-européenne. L'ancienneté et le conservatisme attribués aux sources sanskrites l'invitèrent donc à prendre ces dernières comme comparants et à rechercher des correspondances possibles dans d'autres textes de la sphère indo-européenne. La problématique et la méthode comparative qu'il suivit dans les mariages indo-européens furent les mêmes que celles qu'il avait développées dans le problème romain des flamines majeurs et qui avaient abouti à la reconstruction de l'idéologie tripartite indo-européenne de ig38. À cette exception près, qu'il avait comme a priori sa propre théorie de la trifonctionnalité qu'il essaya alors d'appliquer aux formes de mariages indiens et romains. Pour le reste, le chemin fut le même: partir d'un problème romain, déterminer une structure idéologique indienne, puis calquer cette dernière sur les données romaines.

17 Le seul indianiste fut Alf Hiltebeitel, alors Associate Professor à la George Washington University. Si, dans son compte rendu (History of Religions 5, I980, p.370-372), il accepta l'hypothèse dumézilienne, il releva néanmoins que la pluralité des énoncés normatifs posait problème. Il émit également des doutes sur l'interprétation décontextualisée de G.Dumézil au sujet du mariage svayaṃvara d'Ambā relaté dans le Mahābhārata. Jean Gagé (I902-I986), dans sa recension (Latomus 40, I98I, p.444-445), fut avant tout critique sur la lecture que fit G. Dumézil de l'inscription du vase du Quirinal. Quant à Henri Le Bonniec (I9I5-I994), il ne put guère, dans sa recension (Revue des études latines 58, I980, p.599-6o2), porter son attention sur le domaine indien. S’il écrivit «que pour suivre la démonstration dans ses méandres et ses subtilités, le lecteur doit avoir quelques notions de droit romain ", combien plus le lecteur devait, face à un tel comparatisme, connaître et le sanskrit et la littérature normative de l'Inde ancienne! Enfin, dans son compte rendu (Revue de l'Histoire des religions I97/I, I980, p.92-94), Robert Turcan se contenta d'en résumer le contenu et ne formula aucun avis critique.

18 B. Sergent, Les Indo-Européens. Histoire, langues, mythes, Paris, Payot, I995, p. 225-227; J.P. Mallory and D. Q. Adams, Encyclopedia, p.369-37o; Ch. Malamoud, Féminité de la parole, études sur l'Inde ancienne, Paris, Albin Michel, 2005, p. 26I-27I. 
Dans son introduction intitulée «le problème romain ${ }^{19}$ », G. Dumézil rappela que ce fut Pierre Noailles $^{20}$ (I88I-I943) qui, au printemps I943, l'entraîna dans un comparatisme touchant aux trois formes romaines d'acquisition de la manus, à savoir confarreatio, usus et coemptio, et la trifonctionnalité indo-européenne. Seul l'usus résistait à une classification dans la deuxième fonction. Les tâtonnements de G.Dumézil, exprimés dans des conférences en I952, I955, I958 puis ı970, aboutirent finalement à la rédaction de l'ouvrage de i979. L'approche méthodologique demeurait toujours la même. À partir des données indiennes, il était possible de démontrer que l'usus était héritier de la deuxième fonction de l'idéologie indoeuropéenne et qu'ainsi confirmés, les trois modes de mariages indo-européens pouvaient être recherchés dans les épopées grecque, romaine, indienne et scandinave. À cet ensemble de textes épiques, il convient d'ajouter une étude du récit des mariages successifs des trois filles du Kaisar de Roum, écrit par Firdūsī (940-IO20) dans son $\breve{S} h a \bar{h}$ Nâmeh au x ${ }^{\mathrm{e}}-\mathrm{xI}^{\mathrm{e}}$ s. ap. J.-C., qui fut publiée comme $9^{3 \mathrm{e}}$ esquisse de mythologie en $1995^{21}$. Nous n'aborderons pas ici l'implication de la méthode de mythologie comparée suivie par G.Dumézil dans sa lecture des différents textes grec, romain, indien, iranien et scandinave après qu'il ait restitué les trois modes de mariages romano-indiens. En effet, ce comparatisme mythologique n'est que la résultante de la démonstration de la tripartition des modes de mariages indiens sur laquelle il convient de revenir en premier lieu.

Le comparatisme pratiqué par G. Dumézil demande donc à évaluer le traitement des sources sanskrites qu'il opéra et à partir duquel il confirma non seulement la corrélation possible entre l'usus romain et la deuxième fonction, mais encore la restitution des trois modes romains de mariages héritiers de l'idéologie trifonctionnelle indo-européenne qu'il serait alors également possible de retrouver dans des récits mythologiques et épiques. La première difficulté, et non des moindres d'un point de vue historique, que le savant français avait toujours à lever, était l'absence dans le Rg veda lui-même d'occurrences explicites et claires de cette trifonctionnalité indo-européenne qu'il forgea à partir des sphères culturelles romaine et indienne en I938. Malgré tous ses efforts pour parvenir à déceler ces trois fonctions juxtaposées et hiérarchisées dans le $R g$ veda, les résultats, qu'il publia en ig6r, ne sont nullement probants ${ }^{22}$. Pour les modes de mariages, G. Dumézil fut obligé de

DuméziL, Mariages indo-européens, p. I7-29.

M. Malherbe,LaFacultéde Droit de Bordeaux (I870-I970), Talence, Presses Universitaires de Bordeaux, I996, p.39o-39i.

21 G. Dumézil, Le roman des jumeaux et autres essais. Vingt-cinq esquisses de mythologie (76-IOo), publiées par Joêl H. Grisward, Paris, Gallimard, I995, p. 254-263.

22 G. DumÉzIL, “Les "trois fonctions" dans le $R g$ Veda et les dieux indiens de Mitani», Bulletin de la classe des lettres et des sciences morales et politiques, $5^{\mathrm{e}}$ série, tome 47, Bruxelles, Palais des Académies, I96I, p. 265-298. 
reconnaître à nouveau que les compositions les plus anciennes de l'Inde, versifiées tel le $R g$ veda ou en prose tels les Brāhmaṇa, n'exposaient aucune "théorie raisonnée ${ }^{23}$ » des mariages. Néanmoins, après avoir présenté les textes normatifs des śāstra et des sūtra, il revint sur les occurrences rgvédiques attestant la mention par les poètes de différentes formes de mariages à l'exception évidemment de la forme brāhma. Il contrecarra cette absence en affirmant que «d'une part, nous sommes assurés de l'antiquité de la classe sacerdotale indienne et, d'autre part, l'extrême considération avec laquelle est traitée la brāhmaṇi ${ }^{24}$ ». Ces deux arguments suffisaient, semble-t-il, pour soutenir l'existence historique à la période du $R g$ veda du mode de mariage brāhma. S’il paraît évident, à la lecture du $R g$ veda, qu'au cours du II 'e millénaire avant notre ère, entre I75o et i20o av. J.-C., à l'époque du chalcolithique et de l'âge du Bronze dans le Nord-Ouest de l'Inde, des formes de mariages différentes étaient pratiquées, ces dernières en revanche ne faisaient aucunement l'objet d'une classification idéologique. Mais pour G.Dumézil, il ne faisait aucun doute que cette absence classificatoire était simplement due au genre hymnique et à la matière traitée. Or, ni le genre, ni la finalité hymniques ne peuvent être considérés comme ayant été des obstacles à l'énoncé d'une structure tripartite, bien au contraire. En d'autres termes, là où les compositions indoārya sont les plus anciennes et devraient avoir le mieux conservé un quelconque héritage indo-européen, à l'égal de la langue rgvédique, il n’en est rien. G. Dumézil a donc, comme à son habitude, reporté son attention sur les traités normatifs et l'épopée du Mahābhārata qui sont loin d'avoir l'ancienneté du R g veda.

Dans le domaine du droit indien, il prit, sans justifier son choix, le Mānavadharmaśāstra pour «texte de base ${ }^{25}$ ». Quels furent les critères historiques et juridiques qui l'amenèrent à prendre ce traité normatif, rédigé par un brāhmane lettré assurément plus théoricien que juriste et ne remontant pas au-delà du $\mathrm{II}^{\mathrm{e}} \mathrm{s}$. av. notre ère? Probablement parce qu'il offrait une classification sociale des formes de mariages à la différence de śâstra plus anciens, comme le Gautamadharmasūtra (entre le viI et le $\mathrm{v}^{\mathrm{e}} \mathrm{s}$. av. J.-C), qui ne faisaient aucune corrélation entre les modes de mariages et les classes sociales indiennes ou varna. De fait, G. Dumézil poursuivait un but, celui de démontrer, quoiqu'il en coûtât de la méthode comparative, le bien fondé de sa théorie trifonctionnelle indo-européenne en montrant que dans le domaine juridique des mariages, celle-ci pouvait également s'appliquer à l'Inde

23 Dumézil, Mariages indo-européens, p.3r. A. A. MacDonell et A. B. Keith avaient proposé en leur temps la tripartition suivante des mariages védiques: [I] Consentement mutuel (prājāpatya); [2] Contrepartie (āsura, ārșa, brāhma et daiva); [3] Enlèvement (kṣātra et rākșasa). Mais cette classification ne coïncidait aucunement pour G. Dumézil avec sa théorie trifonctionnelle. MacDonell and Кеiтн, Vedic Index, p. 483. 
et à Rome. S'abstenant de toute étude historico-critique des sources sanskrites et de leur histoire rédactionnelle, il sélectionna les textes les plus à même d'étayer sa théorie. C'est ainsi que son choix se porta avant tout sur le Mānavadharmaśāstra et qu'il imputa aux auteurs indiens des autres traités normatifs un ensemble de confusions classificatoires ${ }^{26}$. Ce procédé lui offrit la liberté de reclasser à son gré les différentes formes de mariages indiens selon les classes sociales en excluant celle des suudra. Le mode de mariage svayamvara était également pour lui, non pas un mode à part entière, mais une correction du mode gāndharva ${ }^{27}$. De fait, au lieu de présenter la diversité des classements opérés par les différentes écoles brāhmaniques du nord et du sud de l'Inde, G. Dumézil préféra gommer les divergences pour ne retenir d'un seul ensemble et une seule classification dont il fut finalement lui-même l'auteur. Il passa donc outre les "quelques divergences entres écoles ${ }^{\mathbf{2 8}}$ », avança que «la classification en huit termes est la plus usuelle, qu'elle comporte cependant quelques variantes qui n'affectent que peu de termes et ne changent rien d'important aux définitions ${ }^{29}$ » et conclut “telle est, constante pour l'essentiel à travers les variantes, la doctrine de l'“Inde classique"30". À ceci s'ajoute le fait qu'il encoda les huit modes de mariages indiens. Dans ces conditions, il était bien difficile à un lecteur non indianiste de suivre sa démonstration et de démêler les chemins tortueux et prédéterminés de son comparatisme.

Pour retracer l'interprétation et la classification de G.Dumézil, il est nécessaire de repartir du Mānavadharmaśāstra. L'auteur de ce traité brāhmanique opta pour un classement de huit formes de mariages dont les appellations renvoient à des divinités et dont la valeur est décroissante: I - brāhma ; 2 - daiva; 3 ; - ārșa ; 4 prājāpatya ; 5 - āsura; 6 -gāndharva; 7-rākșasa; 8 -paiśāca. Nous n’avons pas repris ici la traduction de G.Dumézil dont nous pourrions discuter également les choix sémantiques et syntaxiques. Nous donnons donc ci-après une traduction qui suit de près le texte sanskrit de MnŚ 3.27-34:

“[I] Le don d’une jeune fille, qui a été revêtue [d’une robe] et a été honorée [de parures], à [un homme] ayant étudié la science sacrée et ayant une bonne conduite, [et] que [le père] a lui-même invité, est appelé rite de Brahmā $\|27\|$

[2] Le don d'une jeune fille, qui a été ornée [d'une robe et de parures], à un officiant qui exécute le rituel lors d'un sacrifice correctement accompli, est appelé rite des divinités diurnes (deva) $\|28\|$

\footnotetext{
26 Ibid., p.36-39.

27 Ibid., p.54 et 8 I.

28 Ibid., p. 35.

29 Ibid., p. 35.

30 Ibid., p.39.
} 
[3] Le don d'une jeune fille, selon la règle, après que [son père] eut reçu du prétendant un couple de bovins ${ }^{31}$ ou deux [couples de bovins] conformément à la loi [sacrée], est appelé rite des sages antiques (ṛ̦i) ||29\|

[4] Le don d'une jeune fille [par son père] qui s'adresse [au couple] en ces termes:

"Pratiquez tous deux ensemble le devoir!" et qui a honoré [ainsi le prétendant], est appelé par la tradition rite du Maître des créatures (Prajāpati) ||3o\|

[5] Le don d'une jeune fille à [un homme] qui, par son propre désir [de la posséder], a donné des biens matériels ${ }^{32}$, selon ses moyens, aux parents ainsi qu'à la jeune fille, est appelé rite des divinités nocturnes (asura) ||3i $\mid$

[6] L'union d'une jeune fille et d'un prétendant, issue d'un désir mutuel, doit être considérée comme le [rite] des êtres célestes (gandharva). [Elle] est née du désir érotique [et] a en vue [le plaisir] de l'accouplement sexuel ||32\|

[7] L'enlèvement d'une jeune fille par la force, de la maison [de son père], qui crie et qui pleure, [par un homme] qui a tué ou blessé [ses proches parents] et est entré par effraction, est appelé la manière d'agir des démons nocturnes (rākṣas) ||33||

[8] Quand [un homme] viole à l'écart une [fille] endormie, enivrée ou privée de ses facultés, c'est le plus maléfique des mariages et le plus bas, le huitième [appelé la manière d'agir des] démons cannibales (piśāca) $\|34\|^{\mathbf{3 3}}{ }^{\text {» }}$

31 Vers 300 av. J.-C., Mégasthène fut témoin de ce mode de mariage lors de son séjour dans le Magadha. Strabon, Géographie XV, I,54.

32 En 326 av. J.-C., Aristobule de Cassandreia assista sur la place du marché de Taxila à la vente par des pères pauvres de leurs filles nubiles selon le mode āsura (Strabon, Géographie ı.ı..62). Sur la vente et la revente des épouses selon le mode āsura chez les Kirar, voir notamment l'étude de l'anthropologue Jean-Luc Chambard, “Mariages secondaires et foires aux femmes en Inde centrale », L'Homme, tome i, n' 2, ig6r, p. 5i-88.

ācchādya cārcayitvā ca śrutaśîlavate svayam |

āhūya dānaṃ kanyāyā brāhmo dharmạ̣ prakīrtitaḥ ||27||

yajñe tu vitate samyag ṛtvije karma kurvate |

alan̉kṛtya sutādānaṃ daivaṃ dharmaṃ pracakșate ||28\|

ekaṃ gomithunaṃ dve vā varād ādāya dharmatạ̣ |

kanyāpradānaṃ vidhivad ārṣo dharmah sa ucyate ||29||

sahobhau caratāṃ dharmam iti vācānubhāșya ca |

kanyāpradānam abhyarcya prājāpatyo vidhị̣ smṛtaḥ \|30\|

jñātibhyo draviṇaṃ dattvā kanyāyai caiva śaktitạ̣ |

kanyāpradānaṃ svācchandyād āsuro dharma ucyate \|31\|

icchayānyo'nyasaṃyogaḥ kanyāyāś ca varasya ca |

gāndharvaḥ sa tu vijñeyo maithunyaḥ kāmasaṃbhavạ̣ ||32||

hatvā chittvā ca bhittvā ca krośantīṃ rudantīṃ gṛhāt |

prasahya kanyāharaṇaṃ rākṣaso vidhir ucyate $\|33\|$

suptāṃ mattām pramattāṃ vā raho yatropagacchati |

sa pāpiștho vivāhānāṃ paiśācaś cāșțamo 'dhamaḥ ||34|| 
L’auteur du traité (MnŚ 3.24) rappela que certains hommes avisés (kavi) assignaient aux brāhmanes les quatre premiers, aux kṣatriya le seul rākșasa et aux vaiśya ainsi qu'aux śūdra le seul āsura. Mais, selon lui (MnŚ 3.23-26), les brāhmanes peuvent pratiquer les six premiers, les kșatriya les quatre derniers, les vaiśya et les śūdra les quatre derniers sauf le mode rākșasa. Néanmoins, l'auteur ajouta immédiatement (MnŚ 3.26) que les modes āsura et paiśāca sont adharmiques et qu'en conséquence de quoi, ils ne doivent pas être pratiqués par celui qui respecte le dharma. Quant aux modes gāndharva et rākșasa, ils peuvent être employés par un kṣatriya soit séparément soit ensemble (fig. I).

\begin{tabular}{|c|c|c|}
\hline & $\begin{array}{l}\text { Selon certains kavi } \\
\quad(\mathrm{MnS} S 3.24)\end{array}$ & $\begin{array}{c}\text { Selon l'auteur } \\
\text { (MnŚ 3.23 et 25-26) }\end{array}$ \\
\hline 1 - BRĀHMA & brāhmaṇa & brāhmaṇa \\
\hline 2 - DAIVA & brāhmaṇa & brāhmaṇa \\
\hline 3 - ÂRȘA & brāhmaṇa & brāhmaṇa \\
\hline 4 - PRĀJĀ̄PATYA & brāhmaṇa & brāhmaṇa \\
\hline 5 - ĀSURA & vaiśya / śūdra & brāhmaṇa / kșatriya / vaiśya / śūdra \\
\hline 6 - GĀNDHARVA & $\varnothing$ & brāhmaṇa / kșatriya / vaiśya / śūdra \\
\hline 7 - RĀKȘASA & kșatriya & kșatriya \\
\hline 8 - PAIŚÁĀCA & $\varnothing$ & kṣatriya / vaiśya / śūdra \\
\hline
\end{tabular}

Fig. I. Répartition sociale des mode de mariages dans le Mānavadharmaśāstra

De ce fait, dans cette société ārya idéalisée qui repose sur le dharma et que présenta l'auteur du Mānavadharmaśāstra, les brāhmanes auraient eu la légitimité de se marier selon les modes brāhma, daiva, ārșa, prājāpatya et gāndharva, les kṣatriya selon les modes gāndharva et rākșasa et enfin les vaiśya et les śūdra selon le seul mode gāndharva. Ainsi, l'unique mode de mariage commun à tous les varṇa est celui fondé sur l'amour réciproque ou gāndharva (fig.2).

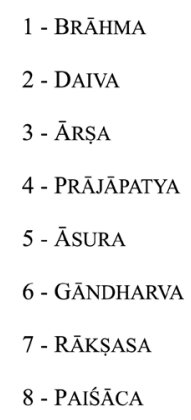

\begin{tabular}{l}
\hline brāhmaṇa \\
brāhmaṇa \\
brāhmaṇa \\
brāhmaṇa \\
interdit (adharmique) \\
brāhmaṇa / kṣatriya/ vaiśya / śūdra \\
kșatriya \\
interdit (adharmique)
\end{tabular}

Fig. 2. Répartition sociale des mode de mariages légaux selon l'auteur du Mānavadharmaśāstra 
Dans sa démonstration, G.Dumézil reclassa ces modes de mariages en fonction de sa théorie de la trifonctionnalité indo-européenne. Pour y parvenir, il dut éliminer le mode paiśāca déclaré adharmique et prohibé par l'auteur du Mānavadharmaśāstra: “L’abominable paiśāca étant écarté, les modes se répartissent en quatre groupes ${ }^{34}$. Mais pourquoi, dans ce cas, écarta-t-il uniquement le mode paiśāca puisque l'auteur du Mānavadharmaśāstra spécifiait clairement que le mode āsura était tout autant adharmique et prohibé? De fait, G.Dumézil conserva le mode āsura, car il correspondait, pour lui, à la troisième fonction. Ainsi opta-t-il pour une classification quaternaire de sept modes de mariages sur huit qu'il coupla avec un abstrait, à savoir [I] pour les modes brāhma, daiva, ārșa, prājāpatya, le don (dāna) gratuit de la fille fait par le père au prétendant, [2] pour le mode rākșasa, le rapt de la fille par la force (prasahya) perpétré par un homme, [3] pour le mode gāndharva, le consentement mutuel fondé sur le seul désir (kāma), et enfin [4] pour le mode āsura, la vente de la fille comme bien matériel (draviṇa) par son père à l'acheteur ${ }^{35}$. Et G. Dumézil de conclure «malgré quelques divergences entre les écoles, les sept modes "acceptés" sont répartis naturellement entre les varṇa ārya: les quatre premiers conviennent particulièrement aux brāhmaṇa, les modes rākșasa et gāndharva aux kṣatriya, le mode āsura aux vaiśya ${ }^{36}$ » (fig. 3).

1 - BRĀHMA
2 - DAIVA
3 - ĀRṢA
4 - PRĀJĀPATYA
7 - RĀKȘASA
6 - GĀNDHARVA
5 - ĀSURA
8 - PAIŚĀCA

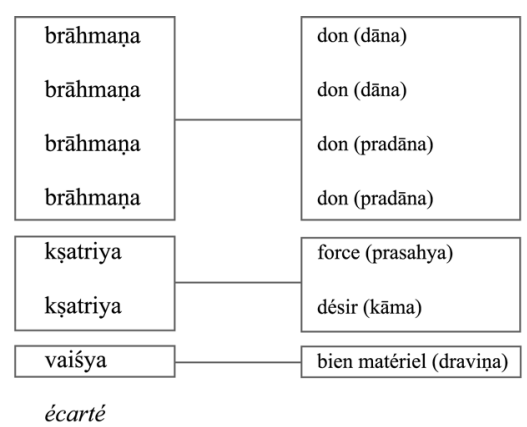

Fig. 3. Classification et répartition sociale des mode de mariages d'après G. Dumézil à partir du Mānavadharmaśāstra et selon sa théorie de la trifonctionnalité indo-européenne de Ig38

Pour le mode āsura, G.Dumézil renvoya son lecteur à l'étude fort datée de Léon Feer (I83o-I902), publiée sous le titre “Le mariage par achat dans l'Inde âryenne» (Journal asiatique I885), alors que Jan Gonda (I905-I99I) avait édité une recherche bien plus rigoureuse sur les modes ārșa et āsura en 1975 (J. Gonda, "Reflections on the Ārșa and Āsura forms of Marriage », in J. Gonda, Selected Studies, vol. IV : History of Ancient Indian Religion, Leiden, E.J.Brill, I975, p.I7I-I85). Mais Dumézil n'appréciait guère J. Gonda.

DumÉzil, Mariages indo-européens, p. 35. 
Or, non seulement le mode āsura était considéré dans son “texte de base» comme adharmique et prohibé, mais encore le mode gāndharva était le seul à être commun à l'ensemble des trois varṇa indo-ārya, voire même aux quatre avec les śūdra, et, reposant sur le seul désir érotique (kāma), ne pouvait être attribué à l'idéologie de la deuxième fonction, relevant de la classe sociale des kṣatriya et incarnant la force guerrière. Ce mode gāndharva correspondait bien mieux à l'idéologie de la troisième fonction, celle de la sexualité et pouvait donc renvoyer à la classe des vaiśya. C'est pourquoi G. Dumézil substitua à la notion de kāma le concept de liberté qu'il attribua au mode gāndharva par le biais d'une unique occurrence, celle de l'épisode de Śakuntalā dans le Mahābhārata (Mbh). Quant au mode paiśāca qu'il écarta, celui-ci aurait pu être assimilé, selon ou non sa théorie des trois péchés du guerrier, à la troisième fonction. Par ailleurs, si G. Dumézil avait pris en considération MnŚ 3.24 - la classification ancienne opérée par des kavi -, il aurait pu effectivement prétendre à une classification tripartite à savoir brāhma, daiva, ārșa, prājāpatya pour les brāhmanes, rākșasa pour les kṣatriya et āsura pour les vaiśya. Mais il perdait alors le bénéfice du gāndharva, de l'union librement consentie, qui lui était indispensable pour justifier le mode usus à Rome. Il est un fait qu'aucun des traités normatifs n'attestait une classification tripartite claire et explicite en fonction des classes sociales indo-ārya qui lui aurait offert l'opportunité d'une correspondance parfaite avec sa théorie de la trifonctionnalité indo-européenne.

L'auteur de l'épisode de Śakuntalā cita expressément en Mbh r.67.8-9 le Mānavadharmaśāstra comme sa source normative des huit modes de mariages. Ceci n'est pas sans poser un problème de datation du récit épique de Śakuntalā qui, en l'occurrence, ne remonterait pas au-delà du $\mathrm{II}^{\mathrm{e}}$ s. av. J.-C. À la lecture du texte épique dont nous donnons la traduction, il est aisé de constater combien la classification sociale des modes de mariages fut intégrée dans le projet littéraire de l'auteur afin de mettre en exergue le mode gāndharva :

“[8] Ainsi, en résumé, huit unions légales sont reconnues par la tradition: brāhma, daiva, ārșa, prājāpatya, āsura [9] gāndharva rākṣasa et paiśāca reconnu comme la huitième. Manu, né de lui-même, a énuméré dans l'ordre ancestral les devoirs de chacun d'entre eux. [Io] Considère les quatre premiers comme de bon augure pour un brāhmane. Les six premiers, sache, ô toi qui es irréprochable, qu'ils sont légitimes pour un kṣatriya. [II] Et, selon la tradition, on dit que, [le mode] des démons nocturnes est pour les chefs claniques et le mode des divinités nocturnes pour les vaiśya et les śūdra. Néanmoins, des cinq derniers, trois sont légitimes et deux sont illégitimes selon la tradition. [I2] Le mode des démons cannibales et le mode des divinités nocturnes ne doivent jamais être pratiqués. Grâce à cette règle, est prescrit par la tradition le chemin du devoir que l'on doit suivre. [I3] Les deux modes des êtres célestes et des démons nocturnes sont légitimes pour un guerrier, ne crains rien. Séparément ou bien ensemble, ils peuvent 
être pratiqués, cela ne fait aucun doute. [I4] Toi, qui es désireuse de moi qui te désire, ô ma belle, tu peux devenir mon épouse par le mariage des êtres célestes ${ }^{37}$.

Ainsi, selon cet auteur, les quatre premiers modes sont-ils légitimes pour un brāhmane, les six premiers pour un kṣatriya, le mode rākṣasa pour un rājan, le mode āsura étant réservé aux vaiśya et aux śūdra. Néanmoins, les interdits, mentionnés par l'auteur et frappant les modes paiśāca et āsura, privent les vaiśya et les śūdra de toute union. Quant au kșatriya, les deux modes de mariages par excellence qui lui reviennent, sont le gāndharva et le rākșasa, pratiqués ensemble ou séparément. Dans son commentaire, G. Dumézil passa sous silence le fait que les kṣatriya avaient accès aux six premiers modes de mariages et ne retint que le seul mode rākșasa pour les chef claniques ou rois qu'il identifia aux kṣatriya eux-mêmes ${ }^{38}$. L'auteur de Śakuntalā énuméra donc bien la liste des huit mariages du Mānavadharmaśāstra sans pour autant les attribuer aux mêmes classes sociales (fig.4).

37 așțāv eva samāsena vivāhā dharmatah smṛtāh | brāhmo daivas tathaivārṣaḥ prājāpatyas tathāsurạ̣ ||8\| gāndharvo rākṣasaś caiva paiśācaś cāșțamaḥ smṛtạ̣| teșāṃ dharmān yathāpūrvaṃ manuḥ svāyaṃbhuvo 'bravīt ||9|| praśastāṃś caturaḥ pūrvān brāhmaṇasyopadhāraya | șaḍ ānupūrvyā kṣatrasya viddhi dharmyān anindite $\|10\|$ rājñāṃ tu rākșaso 'py ukto vițśūdreșv āsuraḥ smṛtaḥ| pañcānāṃ tu trayo dharmyā dvāv adharmyau smṛtāv iha \|11\| paiśācaś cāsuraś caiva na kartavyau kathạ̣ cana| anena vidhinā kāryo dharmasyaișā gatị̣ smṛtā $\|12\|$ gāndharvarākṣasau kṣatre dharmyau tau mā viśañkithāḥ | pṛthag vā yadi vā miśrau kartavyau nātra saṃśayaḥ $\|13\|$ sā tvaṃ mama sakāmasya sakāmā varavarṇini | gāndharveṇa vivāhena bhāryā bhavitum arhasi ||14\| Dumézil, Mariages indo-européens, p.37. Il donna également la traduction de Mbh I.96 dans lequel sont listés les huit modes, sans corrélation avec les classes sociales à part pour le dernier: [I] brāhma, [2] ārșa, [3] āsura [4] rākșasa, [5] paiśāca, [6] gāndharva, [7] ārșa, et [8] svayamıvara pour les seuls rājanya, guerriers d'origine royale. On notera l'absence des modes daiva et prājāpatya, un redoublement du ārșa et l'intégration du svayaṃvara. 


1 - BRĀHMA
2 - DAIVA
3 - ĀRṢA
4 - PRĀJĀPATYA
5 - ĀSURA
6 - GĀNDHARVA
7 - RĀKȘASA
8 - PAIŚĀCA

brāhmaṇa / kșatriya
brāhmaṇa / kṣatriya
brāhmaṇa / kṣatriya
brāhmaṇa / kṣatriya
kṣatriya / vaiśya / śūdra
kșatriya
rājan
$\varnothing$

interdit

interdit

Fig. 4. Répartition sociale des mode de mariages selon Mahābhārata I.67.8-14

Parmi les traités normatifs, seul l'auteur du Baudhayanadharmaśāstra (BauŚ I.II.20.8-I5) mit en corrélation les modes de mariages avec les classes sociales. Il déclara légitime les mariages brāhma, prājāpatya, ārșa et daiva pour les brāhmanes, les modes āsura et rākṣasa pour les kṣatriya, et enfin les mariages gāndharva et paiśāca pour les vaiśya et les śūdra. Au sujet de ces deux derniers, G.Dumézil, qui avait pourtant travaillé à partir des traductions anglaises de ces śāstra publiées dans la collection des Sacred Books of the East, distribua aux vaiśya le mode gāndharva et aux śūdra le paiśāca ${ }^{39}$. Or, dans la phrase pañcamāștamau vaiśyaśūdrānām, les composés copulatifs (dvandva), qui servent souvent dans les traités à l'énumération, au nominatif duel et au génitif pluriel n’ont pas valeur distributive: «Les [deux modes] cinquième et huitième [sont légitimes] pour les vaiśya et les śūdra.» Par ailleurs, en BauŚ ı.II.20.ı6, l'auteur déclara le paiśāca prohibé et le gāndharva accessible au quatre varṇa (fig. 5).

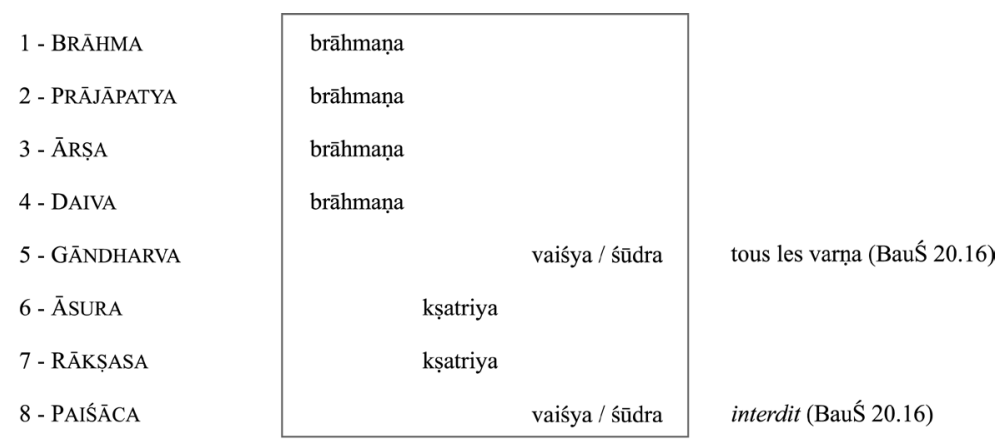

Fig. 5. Répartition sociale des mode de mariages légaux selon Baudhayanadharmaśāstra I.II.20-8-ı6

39 DumÉzil, Mariages indo-européens, p. 36. 
Cette classification propre au Baudhayanadharmaśāstra ( $\mathrm{v}^{\mathrm{e}}-\mathrm{IV}^{\mathrm{e}} \mathrm{s}$. av. J.-C.) et ne correspondant pas à celle du Mānavadharmaśāstra, qui lui est postérieur d'au moins trois siècles, embarrassa G.Dumézil. Il résolut le problème des divergences en déclarant qu'il y avait de la part de son auteur, “évidemment quelque confusion ${ }^{40}$ ». Le mariage gāndharva n'était pas, en effet, attribué aux kṣatriya, mais était un mode légitime pour les vaiśya et les śūdra. Par ailleurs, l'auteur du Baudhayanadharmaśāstra (I.II.20.I6) affirmait, comme celui du Mānavadharmaśāstra le fera en son temps, que certains recommandaient à l'ensemble des varṇa le gāndharva, seul mode à être fondé sur un amour réciproque (gāndharvam apy eke praśamsanti sarveșām snehānugatatvāt). Ce mode de mariage est celui qui fut également conseillé à toutes les classes sociales par Vātsyāyana (III ${ }^{\mathrm{e}} \mathbf{S}$. ap. J.-C.) dans son Kāmasūtra ${ }^{41}$. Aussi, il apparaît que, dans les traités normatifs, les brāhmanes juristes n'ont jamais fait du mode gāndharva une spécificité guerrière. Il est le fruit de la littérature romanesque propres aux kṣatriya exaltant l'amour réciproque là où les jeunes garçons et filles kṣatriya faisaient l'objet de mariages arrangés afin d'assurer des alliances claniques et où les jeunes filles risquaient de n'obtenir que le difficile statut de coépouse.

Pour le reste, les autres traités normatifs, à savoir, pour les périodes anciennes, le Gautamadharmasūtra (6oo-40o av. J.-C.), 1'Āśvalāyanagthyasūtra (5oo av. J.-C.), l'Āpastambadharmasūtra (45o-35o av. J.-C.), le Vāsișthadharmaśāstra (3oo-Ioo av. J.-C.) et, pour les périodes plus tardives, le Vaiṣnavadharmaśāstra (200-3oo ap. J.-C.) ainsi que le Nāradīyadharmaśāstra (3oo-40o ap. J.-C.) n’offraient aucune répartition sociale des formes de mariages. Il ressort que dans ces traités normatifs recouvrant plus de mille ans d'histoire de la société indo-ārya, cette classification sociale ne fut aucunement fondamentale et qu'aucun schéma tripartite ou autre n'ait prévalu. Pour les brāhmanes, auteurs de ces traités normatifs, la priorité résidait avant tout dans l'énumération de cinq à huit formes de mariages classées selon un ordre de préséance divine. Ils tenaient, de ce fait, en haute estime le mode brāhma, c'est-à-dire le mariage arrangé entre un père et un prétendant, le père faisant alors don à son futur gendre de sa fille ou plus exactement de l'autorité paternelle qu'il exerçait sur sa fille. Et ces mêmes brāhmanes rejetaient sans équivoque le paiśāca. Pour avoir êté unanimement condamné dans la société indo-ārya, le mariage forcé par le viol de la jeune fille ne devait pas être chose rare. L'auteur du Mānavadharmaśāstra, qui écrivait avant tout pour la classe sociale brāhmanique et la défense de ses droits, n'hésita pas à promulguer un châtiment corporel pour le violeur d'une jeune fille, qu'il fût kṣatriya, vaiśya ou śūdra (MnŚ 8.364). Quant au brāhmane qui commettait le même crime, il ne se voyait frappé que d'une simple

\footnotetext{
$40 \quad$ Ibid., p.36.

41 Kāmasūtra 3.5.3o.
} 
amende (MnŚ 8.378). Quoi qu'il en soit, dans l'état actuel des traités normatifs brāhmaniques, la première répartition des modes de mariages en fonction des varṇa ne remonte pas avant le $v^{\mathrm{e}} \mathrm{s}$. av. J.-C. dans le sud de l'Inde (fig. 6).

G.Dumézil avait parfaitement conscience de la fragilité de sa démonstration basée sur la classification sociale des modes de mariages indiens dont l'ancienneté ne pouvait être védique. C'est pourquoi il affirma que «le système des varṇa, avec sa rigueur, n'est pas chose primitive dans l'Inde. Les plus anciennes sociétés arya, la société indo-iranienne, n'avaient sans doute pas encore de classes sociales étanches, endogames, strictement délimitées ${ }^{42}$ ». Il justifia donc sa restitution tripartite des mariages indiens, et a fortiori indo-européens, en transférant de la sphère sociale au domaine idéologique cette tripartition: "Ce qui était rigoureusement triparti, trifonctionnel, c'étaient les spéculations idéologiques, y compris un modèle de société éventuellement différent de la réalité. Or le système des mariages pouvait exister dès ce temps d'avant les varṇa ${ }^{43}$. . Cet argument suffisait, à ses yeux, pour imposer une classification tripartite des mariages indiens, à savoir un mode fondé sur le prestige du sacré, deux modes fondés respectivement sur la force et la liberté et enfin un mode fondé sur l'intérêt matériel ${ }^{44}$. Une fois ce passage idéologique par l'Inde terminé, il pouvait alors revenir au monde romain et à l'explication de l'usus. Cependant cette restitution du tableau triparti des mariages indiens fut réalisée à partir de la classification sociale de ces mêmes mariages telle qu'elle avait été opérée et exposée par l'auteur du Mānavadharmaśāstra. Aussi, suivant son argument de la spéculation idéologique indo-ārya, G.Dumézil aurait pu s'abstenir de passer par cette classification sociale post-védique, et donc historique, et ne restituer qu'un tableau tripartite idéologique à partir des seules définitions données par l'auteur du traité indien. Il aurait ainsi pu établir trois modes de mariages indiens (brāhma, rākṣasa et āsura) renvoyant aux trois fonctions indo-européennes à savoir le don sacré, le rapt et l'achat. Mais une telle restitution le privait encore une fois du mariage gāndharva.

\footnotetext{
42 DumézIL, Mariages indo-européens, p.44.

43 Ibid., p.44.

44 Ibid., p. 45.
} 


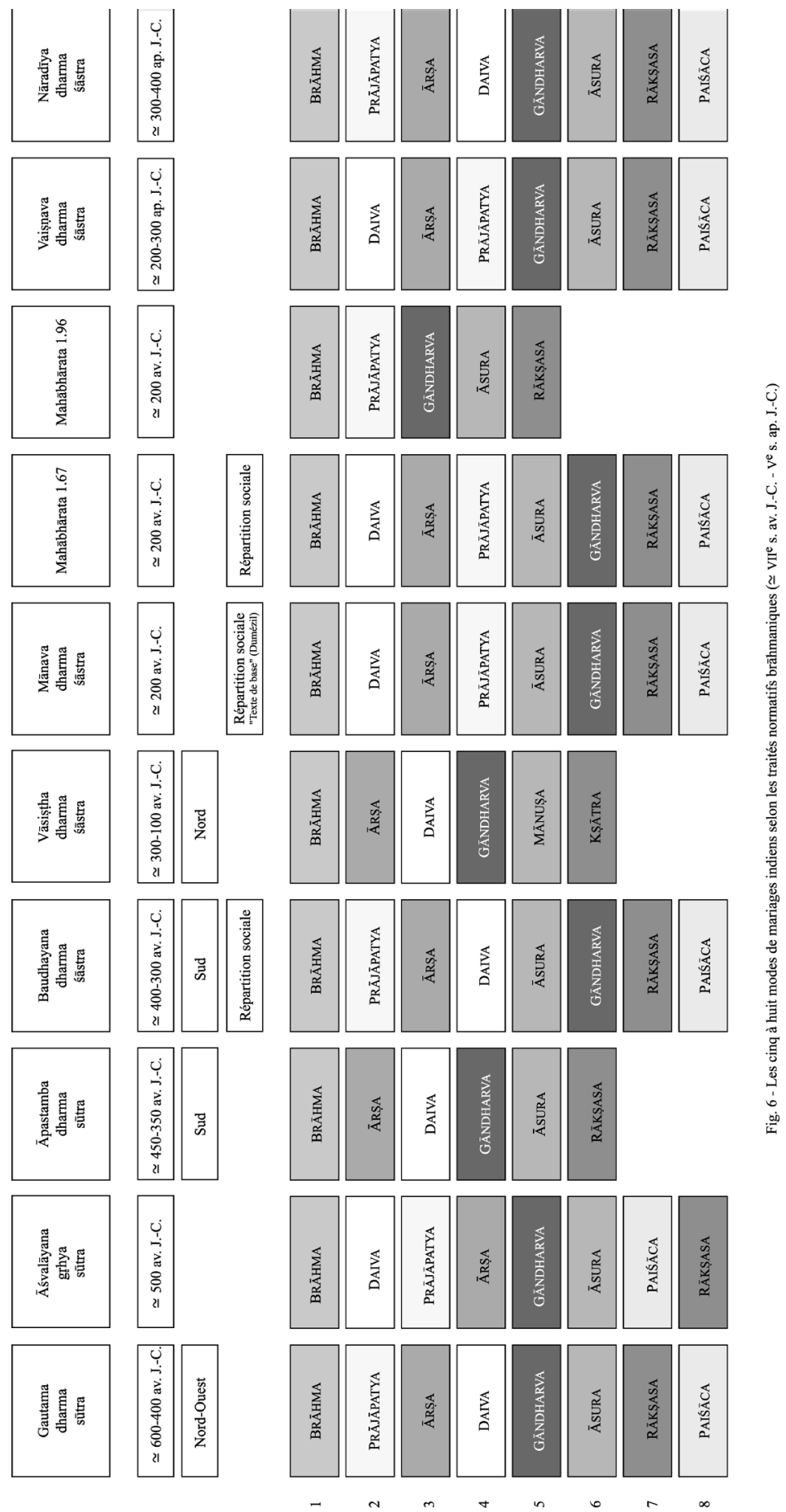


Par ailleurs, cette double approche de la trifonctionnalité des mariages indiens, l'une sociale, l'autre spéculative, qui atteste combien sa méthode de restitution et sa théorie de la trifonctionnalité indo-européenne étaient encore mal différenciées en I979, entremêle les deux théories successives qu'il énonça au cours de sa carrière intellectuelle. De I939 à I949, la première théorie de la trifonctionnalité reposait selon lui sur une réalité sociale historiquement attestée au moins en Inde, voire dans le monde indo-iranien. Cette hypothèse fut grandement influencée par les travaux des sociologues sur la classification collective, notamment ceux d'Émile Durkheim (1858-I9I7) et Marcel Mauss (1872-I95o) ${ }^{45}$. La seconde théorie fut consécutive au revers que G.Dumézil essuya lorsqu'il tenta de démontrer en i94I, dans Jupiter Mars Quirinus, que les trois tribus légendaires de Rome (Ramnes, Luceres, Tities) étaient les équivalents mythiques de classes fonctionnelles réelles. Les critiques à l'encontre de cette lecture des trois tribus légendaires de Rome l'obligèrent à reporter sa théorie trifonctionnelle sociale dans le domaine de la spéculation intellectuelle qui, dès lors, était considérée comme autonome dans chacune des cultures héritières de la langue et de la pensée proto-indo-européennes et n'avait donc plus nécessité de calquer une réalité sociale. Cette avancée théorique ouvrit G.Dumézil à une plus grande liberté, car elle lui permettait alors d'investir l'ensemble des textes mythologiques sans plus être tributaire des classes sociales historiquement attestées. Néanmoins, dans le cas des formes de mariages indiens, sa démonstration reposa en premier lieu sur son approche trifonctionnelle d'avant I949. Dans le cas de l'Inde, il avait, en effet, tout le loisir de ne pas s'affranchir d'une réalité sociale historique. Malheureusement, les classifications sociales des traités normatifs ne correspondaient aucunement à ce qu'il souhaitait démontrer. En effet, ni le mode gāndharva, ni le mode āsura n'étaient spécifiques respectivement aux kṣatriya et aux vaiśya. Ceci l'obligea donc à poursuivre son raisonnement avec une approche purement spéculative de la trifonctionnalité. G. Dumézil ne tira donc pas seulement l'usus romain vers la deuxième fonction à l'aide d'un

45 “Mais c'est surtout à travers les mythologies que l'on voit apparaître, d'une manière presque ostensible, des méthodes de classement tout à fait analogues à celles des Australiens ou des Indiens de l'Amérique du Nord. Chaque mythologie est, au fond, une classification, mais qui emprunte ses principes à des croyances religieuses, et non pas à des notions scientifiques. Les panthéons bien organisés se partagent la nature, tout comme ailleurs les clans se partagent l'univers. Ainsi l'Inde répartit les choses, en même temps que leurs dieux, entre les trois mondes du ciel, de l'atmosphère et de la terre, tout comme les Chinois classent tous les êtres suivant les deux principes fondamentaux du yang et du yin. Attribuer telles ou telles choses naturelles à un dieu, revient à les grouper sous une même rubrique génétique, à les ranger dans une même classe». É. Durkheim et M. Mauss, “De quelques formes de classification. Contribution à l'étude des représentations collectives ", Année sociologique 6, I9oI-I9o2, p.4I. 
comparatisme analogique orienté et forcé avec le mode gāndharva, il tira d'abord le mode gāndharva lui-même vers la deuxième fonction. Comme souvent dans son comparatisme, lorsque se dressait devant lui un obstacle de taille, la solution, qui lui permettait de le franchir ou de le contourner, et ainsi de maintenir sa théorie trifonctionnelle indo-européenne, résidait dans le dédoublement des fonctions. En dédoublant la deuxième fonction et en assignant le mode gāndharva aux seuls kṣatriya, il pouvait résoudre le problème posé par l'usus. Puisque, dans le $R g$ veda, le dieu guerrier Indra était proclamé autonome (svadhā), que, dans le Mahābhārata, Śakuntalā supposait être libre ou maîtresse d'elle-même (radi cātmā prabhur mama) pour pouvoir se donner au roi Duhșanta par le mode gāndharva, et que le juriste Gaius (I20-I8o ap. J.-C.) faisait mémoire d'une des lois des Douze Tables (ve s. av. J.-C.) selon laquelle toute femme était libre de rompre le mariage durant sa première année en s'absentant durant trois jours consécutifs, il ne faisait plus aucun doute que le mode gāndharva correspondait à l'usus romain et que ce dernier pouvait alors reprendre sa place dans le «limpide tableau trifonctionnel ${ }^{46}$ ', c'est-à-dire dans la case réservée à la deuxième fonction (fig. 7). Force est de constater que dans l'esquisse posthume des mariages des trois filles du Kaisar de Roum, la théorie de la classification idéologique trifonctionnelle indo-européenne des mariages était acquise et définitive. Plus encore, G.Dumézil recula aux temps védiques le travail classificatoire des modes de mariages qu'il attribua alors à des “liturgistes indiens », auteurs d'une théorie des formes de mariages créée à partir d'une structure tripartite. Les “procédures matrimoniales» étaient dès lors proprement védiques ${ }^{47}$.

De ces remarques méthodologiques il ressort que le comparatisme de G.Dumézil avait la faiblesse de passer outre toute approche historico-critique des sources textuelles et de vouloir systématiquement retrouver, dans des textes de périodes différentes composés dans des contextes historiques différents en des lieux géographiques différents, une structure archétypale prédéfinie qu'il se donnait la liberté d'élargir et de modifier au gré des textes étudiés ${ }^{48}$, mettant alors ces modifications au compte d'évolutions préhistoriques ou historiques. Depuis ses recherches doctorales, l'Inde a toujours représenté pour G.Dumézil une

46 Dumézil, Mariages indo-européens, p.4I. Quant au rapprochement qu'il fit entre l'usurpatio trinoctii romaine et le trirātra indien, Charles Malamoud eut raison de redire, en 2005, qu'ils n'avaient rien en commun et que le trirātra indien relevait d'un rite de consécration du mariage (Malamoud, Féminité de la parole, p. 266). DumÉzil, Le roman des jumeaux, p. 254; 26o et 26I.

48 Depuis, l'anthropologue oxonien Nick J.Allen proposa une quatrième fonction dédoublée, positive et négative. L’helléniste Pierre Sauzeau avança également une théorie de l'idéologie indo-européenne, non plus fondée sur une trifonctionnalité, mais sur une quadrifonctionnalité. 
nébuleuse où des structures sociales et idéologiques indo-européennes furent les mieux conservées, une nébuleuse où les sources textuelles sanskrites, difficilement datables, offraient une grande liberté anhistorique, une nébuleuse, enfin, par laquelle il était toujours indispensable de passer avant de s'appliquer à l'étude des autres sphères culturelles indo-européennes. Or, dans le cas présent, les strates qu'il dégagea dans le monde indien ne l'ont pas été grâce à un outillage méthodologique adéquat, mais à partir d'un comparatisme orienté dont l'objectif premier était de faire entrer l'usus romain dans le cadre de la deuxième fonction. G. Dumézil a donc sélectionné les textes indiens en fonction de sa théorie, extirpé certaines données normatives de leur contexte social et historique précis pour les classifier à nouveau librement, et laissé de côté les éléments inopportuns. Il pouvait alors y calquer la structure tripartite sociale ou idéologique qu'il avait lui-même définie dès I938 et dont il s'évertuait à montrer, souvent en perdant son lecteur dans les méandres de sa pensée reformulée, qu'elle ne relevait en rien d'un «mirage $\mathrm{e}^{49}$ » Cette détermination à prouver la véridicité de sa théorie était d'autant plus forte qu'elle l'amenait régulièrement à énoncer de “profondes leçons de méthodes ${ }^{50}$ ", selon les termes de Claude Lévi-Strauss (I9o8-2009), qui la justifiaient en partie ${ }^{51}$.

Georges Dumézil était tout à fait conscient de la fragilité de sa théorie de l'idéologie trifonctionnelle indo-européenne pour laquelle il espérait néanmoins une certaine pérennité, au moins jusqu'à sa mort ${ }^{52}$. En 1979, il ne lui était plus guère possible de repenser sa théorie structurale, comme il l'avoua à Didier Éribon en I986: “Si j’ai eu tort, elle [ma théorie] aura eu une fonction, elle m’aura amusé. De toute façon, aujourd'hui, il est trop tard pour la refaire, je ne peux plus lui échapper ${ }^{53}$. „ Aussi, à réévaluer les procédés de sa démarche comparative dans le

G. Dumézil, Entretiens avec Didier Eribon, Paris, Gallimard, I987, p. I82.

50 Cl. Lévi-Strauss, "Georges Dumézil: Les Dieux des Germains, collection "Mythes et Religions" », Annales. Économies, Sociétés, Civilisations, $7^{\mathrm{e}}$ année, n5, 1962, p. 998.

51 “L'historien des religions doit, comme tout historien, être docile au document. Avant de se demander quel élément, gros ou menu, il peut en extraire au service d'une thèse, il doit les lire et les relire, s'en pénétrer passivement, réceptivement, en ayant soin de laisser en place, chacun à sa place, tous les éléments, ceux qui obéissent et ceux qui lui résistent. Si l'on s'astreint à cette hygiène, on apprend vite qu'il y a mieux à faire, avec de tels textes, que de les détruire pour insérer dans d'autres constructions quelques débris arrachés à leurs ruines: il y a d'abord à comprendre leur propre structure, la raison qui justifie le rassemblement de leurs éléments, y compris les plus particuliers, les plus bizarres. Ce qu'on perd alors du côté de ce qui semblait être de l'histoire, on le regagne en théologie, en intelligence de la pensée religieuse sous-jacente aux documents. » G. DumÉziL, Les dieux des Germains, Paris, PuF, I959, p. 2 I. 
domaine indien ${ }^{54}$ qui oblige de relire les sources textuelles utilisées et de repérer les éléments retenus et ceux délaissés, à considérer les libertés ascientifiques qu'il s'accorda pour agencer ces mêmes éléments indiens selon une structure archétypale prédéfinie avant d'opérer un transfert culturel et structural dans le domaine romain, il convient de reconnaître que les résultats de son étude comparée des mariages indo-européens n'emporte nullement la conviction. Sa tentative de calquer ses théories trifonctionnelles sociale et idéologique indo-européennes sur les modes de mariages indiens fut assurément un point de départ méthodologique voué à l'échec. Néanmoins, une fois reconnu, cet échec offre aujourd'hui aux indianistes comme aux romanistes l'opportunité de passer outre la théorie dumézilienne des mariages indo-européens et de trouver d'autres facteurs sociétaux et idéologiques à l'origine des différentes formes de mariages qui furent pratiquées dans l'Inde et la Rome antiques.

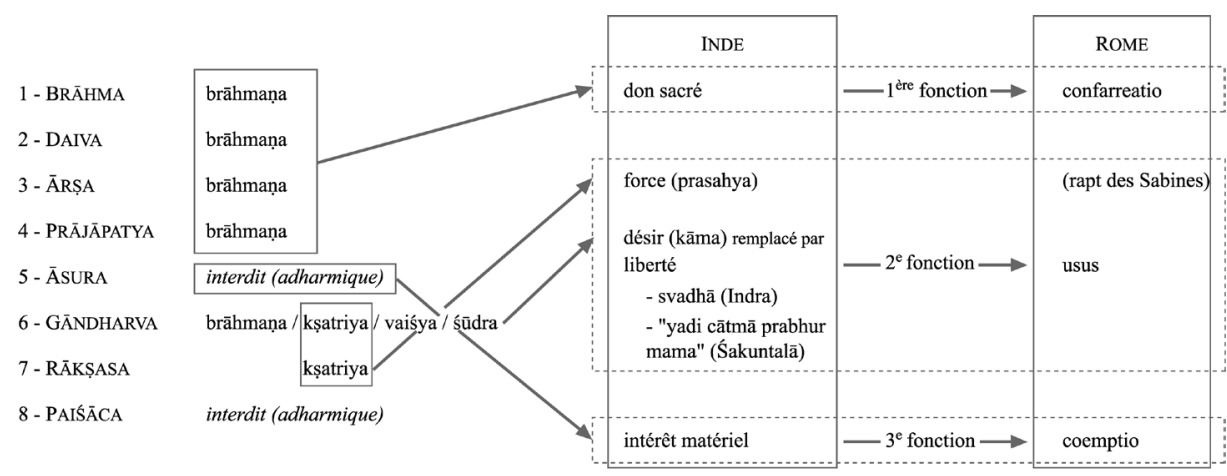

Fig. 7. Répartition sociale des mode de mariages légaux du texte de base (Mānavadharmaśāstra), selon la théorie de la trifonctionnalité indo-européenne de I938, appliquée aux mariages romains par G. Dumézil

\author{
Guillaume Ducœur \\ Maître de conférences, \\ Institut d'histoire des religions \\ Faculté des sciences historiques, \\ Université de Strasbourg \\ gducoeur@unistra.fr
}

54 Nous avons déjà montré combien son repérage de l'idéologie trifonctionnelle dans des sources bouddhiques était irrecevable (G. Duccur, "Georges Dumézil et "le Buddha hésitant” ", in G. TALlet et Ch. Zivie-Coche (éd.), Le Myrte et la rose. Mélanges offerts à Françoise Dunand par ses élèves, collègues et amis, tome 2, сеNiм 9, Montpellier, 2014, p. 199-209). 